\title{
Consumo de energía en la industria siderúrgica mexicana'
}

\author{
A. Elizalde Baltierra, J. García Peláez, L. López Panadero, M. Bauer Ephrussi y J. Quintanilla Martínez \\ Programa Universitario de Energía, UNAM \\ Email:puenergy@servidor.unam.mx
}

(recibido: febrero, 1997; aceptado: febrero, 1998)

\begin{abstract}
Resumen
Se presenta un panorama actual de la industria siderúrgica mexicana. Se analiza el consumo de energía en el sector por fuente de energía (exógena y endógena) y por proceso siderúrgico (convertidor básico al oxígeno y horno eléctrico de arco). Se muestra además el consumo específico de energía en la industria siderúrgica por tipo de empresa (integrada y semi-integrada) y por proceso siderúrgico (convertidor básico al oxígeno y horno eléctrico de arco). Finalmente, se sitúa a nivel internacional al sector, mediante comparaciones de consumo específico de energía, consumo específico de coque en el alto horno, participación del horno Siemens Martin y grado de utilización de colada continua.
\end{abstract}

\section{Abstract}

An overview of the mexican iron and steel industry is presented, including the analysis of the sector's energy consumption by energy source (exogenous and endogenous) and by siderurgical process (basic oxygen conversion and electric arc furnace). The specific industry's energy consumption is shown, by both kind of enterprise (integrated and semi-integrated) and by siderurgical process (BOF and EAF). Finally, its international standing is examined in terms of overall specific energy consumption, specific coque consumption in high furnace, Siemens Martin furnace participation, and utilization degree of continuous casting.

\section{Introducción}

Como en otros países, la industria siderúrgica en México está compuesta por un grupo reducido de empresas integradas, un número mayor de empresas semi-integradas o acerías y un grupo más numeroso de empresas relaminadoras. Las tecnologías empleadas han sido importadas en su mayoría, sin embargo, existe una contribución mexicana importante: el proceso de reducción directa para producir fierro esponja.

El suministro de energéticos proviene predominantemente de las empresas paraestatales: Petróleos Mexicanos (PEMEX), Comisión Federal de Electricidad (CFE) y Luz y Fuerza del Centro (LFC), aunque el autoabastecimiento eléctrico y la cogeneración están presentes. Bajo la presión de la demanda interna y la posibilidad de incrementar su mercado de exportaciones debido a la apertura de la economía, este sector industrial, ahora completamente privado, está pasando por un proceso de expansión y modernización; no obstante, el análisis de la intensidad energética presenta problemas, especialmente en los últimos años, debido a las fluctuaciones de la economía.

Las principales fuentes del presente trabajo son: Cuentas nacionales, Balance nacional de energía, datos estadísticos y publicaciones de la Cámara Nacional de la Industria del Hierro y del Acero (CANACERO), así como las respuestas a la encuesta dirigida por el Programa Universtario de Energía (PUE) a la mayoría de las compañías del sector siderúrgico (apéndice).

1 Trabajo auspiciado por la Dirección General de la Energía (DG XVII), Comisión Europea, red COPED. 


\section{Energía: fuentes, consumo específico e intensidad}

Consumo de energía en el sector

Antes de iniciar con el análisis energético del sector, es importante hacer hincapié en la forma en la que se presentan los datos de consumos de energía. En relación con el consumo de electricidad, éste se presenta como electricidad primaria, o sea, la energía primaria necesaria para la producción de la electricidad que la industria siderúrgica compra al Sector Eléctrico Nacional (SEN). Por lo tanto, a partir de esta última se determina la energía primaria empleando un coeficiente, corres. pondiente a una eficiencia promedio de conversión de $33.5 \%$. De igual forma, en la conversión de los datos sobre coque a datos expresados en carbón primario, se multiplicó el consumo de coque por un factor de 1.3 (Ross, 1987). Estas conversiones a energía primaria nos permiten mostrar los consumos en términos de fuentes primarias, así como calcular los consumos específicos y la intensidad energética con base en normativas internacionales que hacen posible la comparación de los resultados.

En 1994, la industria siderúrgica fue responsable de $16 \%$ del consumo energético industrial en el país. Fue el mayor consumidor, seguido por la industria petroquímica de PEMEX (15.6\%). Los energéticos empleados fueron: gas natural (36.3\%), carbón primario (32.9\%), electricidad primaria (22.9\%), combustóleo (7.4\%), y otros que incluyen gas LP y diesel $(0.5 \%)$. Estos datos hablan por sí mismos de la importancia del análisis energético del sector. a) Por fuente de energía

Las fuentes de energía usadas en instalaciones siderúrgicas pueden clasificarse en fuentes exógenas, compradas fuera de la planta, y en fuentes endógenas, generadas en diversas partes del proceso siderúrgico. Uno de los objetivos del mejoramiento de la eficiencia energética es ampliar lo más posible el uso de las fuentes endógenas y por ende, disminuir las necesidades de energía comprada.

\section{Fuentes exógenas}

Las principales fuentes exógenas de energía para la fabricación de acero son el carbón primario empleado para producir coque, el combustóleo, la electricidad primaria y el gas natural. El consumo de energía primaria en la industria siderúrgica nacional por fuentes exógenas de 1985 a 1994 se presenta en la tabla 1 , de acuerdo con los balances nacionales de energía publicados por la Secretaría de Energía, Minas e Industria Paraestatal (SEMIP), hoy Secretaría de Energía. Además, en la tabla 2 se presentan los consumos de energía secundaria tomando en cuenta a la electricidad como secundaria, tal como la industria la compra al SEN, y al carbón primario expresado en términos secundarios como coque. Los datos relativos a la electricidad se refieren a la comprada al SEN y no se incluye el autoabastecimiento, dado que se genera en gran medida con fuentes endógenas. En las tablas se observa al año 1986 como el de menor consumo debido principalmente al cierre de FUMOSA. Entre 1988 y 1993 se aprecia una ligera reducción del consumo aun cuando la producción de acero sufre un incremento de $18 \%$ durante este periodo.

Tabla 1. Industria siderúrgica nacional: consumo de energía primaria por fuentes exógenas, 1985-19994

\begin{tabular}{|c|c|c|c|c|c|c|}
\hline \multirow{2}{*}{ Año } & \multicolumn{6}{|c|}{ Petacalorías } \\
\hline & Combustóleo & Electricidad primaria** & Carbón primario & Gas natural & Otros $* * *$ & Total \\
\hline 1985 & 2.533 & 11.412 & 25.150 & 19.096 & 1.919 & 60.110 \\
\hline 1986 & 2.347 & 11.728 & 19.629 & 17.918 & 1.812 & 53.434 \\
\hline 1987 & 2.517 & 11.585 & 21.780 & 20.026 & 1.946 & 57.854 \\
\hline 1988 & 5.783 & 19.884 & 16.714 & 25.404 & 0.930 & 68.715 \\
\hline 1989 & 5.271 & 16.146 & 17.506 & 26.196 & 0.782 & 65.901 \\
\hline 1990 & 6.550 & 20.725 & 19.091 & 21.011 & 0.507 & 67.884 \\
\hline 1991 & 6.204 & 17.015 & 16.636 & 20.699 & 0.385 & 60.939 \\
\hline 1992 & 3.651 & 17.776 & 17.581 & 21.128 & 0.316 & 60.452 \\
\hline 1993 & 4.331 & 13.403 & 19.222 & 21.204 & 0.294 & 58.454 \\
\hline 1994* & 4.830 & 14.964 & 21.434 & 23.645 & 0.328 & 65.184 \\
\hline
\end{tabular}


DOI: http://dx.doi.org/10.22201/fi.25940732e.1998.01.004

A. Elizalde B., J. García P., L. López P., M. Bauer E. y J. Quintanilla M.

Tabla 2. Industria siderúrgica nacional: consumo de energía (secundaria) por fuentes exógenas, 1985-1994

\begin{tabular}{|c|c|c|c|c|c|c|}
\hline \multirow[t]{2}{*}{ Año } & \multicolumn{6}{|c|}{ Petacalorías } \\
\hline & Combustóleo & Electricidad** & Coque & Gas natural & Otros*** & Total \\
\hline 1985 & 2.533 & 3.823 & 19.346 & 19.096 & 1.919 & 46.717 \\
\hline 1986 & 2.347 & 3.329 & 15.099 & 17.918 & 1.812 & 41.105 \\
\hline 1987 & 2.517 & 3.881 & 16.754 & 20.026 & 1.946 & 45.124 \\
\hline 1988 & 5.783 & 6.661 & 12.857 & 25.404 & 0.930 & 51.635 \\
\hline 1989 & 5.271 & 5.409 & 13.466 & 26.196 & 0.782 & 51.124 \\
\hline 1990 & 6.550 & 6.943 & 14.685 & 21.011 & 0.507 & 49.696 \\
\hline 1991 & 6.204 & 5.700 & 12.797 & 20.699 & 0.385 & 45.785 \\
\hline 1992 & 3.651 & 5.955 & 13.524 & 21.128 & 0.316 & 44.574 \\
\hline 1993 & 4.331 & 4.490 & 14.786 & 21.204 & 0.294 & 45.105 \\
\hline 1994* & 4.830 & 5.007 & 16.488 & 23.645 & 0.328 & 50.298 \\
\hline
\end{tabular}

${ }^{*}$ Cifras preliminares ${ }^{* *}$ No incluye autoabastecimiento *** Incluye gas LP y diesel. Fuente: balances nacionales de energia, 1988-1994, Secretaria de Energia.

Por otro lado, en la tabla 3 se muestra el consumo de de acuerdo con los datos reportados en la encuesta del PUE energía primaria por fuentes exógenas, en empresas inte- (apéndice). Asimismo, en la tabla 4 se muestran estos consumos gradas y en un grupo representativo de semi-integradas, en términos secundarios.

Tabla 3. Consumo de energía primaria por fuentes exógenas, en empresas integradas y en un grupo representativo de semi-integradas 1988-1994

Empresas integradas

\begin{tabular}{|c|c|c|c|c|c|c|}
\hline \multirow{2}{*}{ Año } & \multicolumn{6}{|c|}{ Petacalorías } \\
\hline & Combustóleo & Gas natural & Carbón primario & Electricidad primaria * & Otros ** & Total \\
\hline 1988 & 4.211 & 14.488 & 17.909 & 8.166 & 0.602 & 45.377 \\
\hline 1989 & 3.939 & 15.366 & 16.028 & 9.212 & 0.587 & 45.132 \\
\hline 1990 & 4.232 & 16.662 & 17.259 & 10.372 & 0.980 & 49.503 \\
\hline 1991 & 3.471 & 17.072 & 14.311 & 10.115 & 0.081 & 45.051 \\
\hline 1992 & 3.469 & 17.256 & 14.719 & 9.588 & 0.010 & 45.042 \\
\hline 1993 & 4.219 & 18.448 & 14.606 & 10.359 & 0.000 & 47.633 \\
\hline 1994 & 3.662 & 19.810 & 16.071 & 11.621 & 0.000 & 51.163 \\
\hline
\end{tabular}

Grupo representativo de empresas semi-integradas

\begin{tabular}{|c|c|c|c|c|c|c|}
\hline \multirow{2}{*}{ Año } & \multicolumn{6}{|c|}{ Petacalorías } \\
\hline & Combustóleo & Gas natural & Carbón primario & Electricidad primaria * & Otros ** & Total \\
\hline 1988 & 0.000 & 0.163 & 0.014 & 0.604 & 0.000 & 0.781 \\
\hline 1989 & 0.000 & 0.170 & 0.014 & 0.704 & 0.000 & 0.888 \\
\hline 1990 & 0.013 & 0.154 & 0.014 & 0.730 & 0.000 & 0.911 \\
\hline 1991 & 0.092 & 0.191 & 0.013 & 0.751 & 0.000 & 1.047 \\
\hline 1992 & 0.077 & 0.198 & 0.000 & 0.892 & 0.225 & 1.393 \\
\hline 1993 & 0.118 & 0.164 & 0.009 & 1.123 & 0.126 & 1.540 \\
\hline 1994 & 0.156 & 0.182 & 0.000 & 1.486 & 0.135 & 1.959 \\
\hline
\end{tabular}


Tabla 4. Consumo de energía (secundaria) por fuentes exógenas, en empresas integradas y en un grupo representativo de semi-integradas, 1988-1994

Empresas integradas

\begin{tabular}{|c|c|c|c|c|c|c|}
\hline \multirow{2}{*}{ Año } & \multicolumn{6}{|c|}{ Petacalorías } \\
\hline & Combustóleo & Gas natural & Coque & Electricidad * & Otros ** & Total \\
\hline 1988 & 4.211 & 14.488 & 13.776 & 2.736 & 0.602 & 35.814 \\
\hline 1989 & 3.939 & 15.366 & 12.329 & 3.086 & 0.587 & 35.307 \\
\hline 1990 & 4.232 & 16.662 & 13.276 & 3.475 & 0.980 & 38.623 \\
\hline 1991 & 3.471 & 17.072 & 11.009 & 3.389 & 0.081 & 35.022 \\
\hline 1992 & 3.469 & 17.256 & 11.322 & 3.212 & 0.010 & 35.270 \\
\hline 1993 & 4.219 & 18.448 & 11.235 & 3.470 & 0.000 & 37.373 \\
\hline 1994 & 3.662 & 19.810 & 12.362 & 3.893 & 0.000 & 39.727 \\
\hline
\end{tabular}

Grupo representativo de empresas semi-integradas

\begin{tabular}{|c|c|c|c|c|c|c|}
\hline \multirow{2}{*}{ Año } & \multicolumn{6}{|c|}{ Petacalorías } \\
\hline & Combustóleo & Gas natural & Coque & Electricidad * & Otros ** & Total \\
\hline 1988 & 0.000 & 0.163 & 0.011 & 0.202 & 0.000 & 0.376 \\
\hline 1989 & 0.000 & 0.170 & 0.011 & 0.236 & 0.000 & 0.416 \\
\hline 1990 & 0.013 & 0.154 & 0.010 & 0.245 & 0.000 & 0.422 \\
\hline 1991 & 0.092 & 0.191 & 0.010 & 0.252 & 0.000 & 0.545 \\
\hline 1992 & 0.077 & 0.198 & 0.000 & 0.299 & 0.225 & 0.799 \\
\hline 1993 & 0.118 & 0.164 & 0.007 & 0.376 & 0.126 & 0.791 \\
\hline 1994 & 0.156 & 0.182 & 0.000 & 0.498 & 0.135 & 0.971 \\
\hline
\end{tabular}

*No incluye autoabastecimiento ** Incluye gas LP y diesel Fuente: encuesta del PUE

El consumo total de energía primaria en empresas integradas presentó un incremento de $11 \%$ entre 1988 y 1994. En particular, resalta el incremento mostrado por la electricidad primaria y el gas natural: 42 y $37 \%$, respectivamente. Por el contrario, el consumo de carbón primario se redujo en $10.3 \%$. En 1994 , los principales energéticos empleados en las empresas integradas fueron el gas natural (38.7\%) y el carbón primario (31.4\%), quedando un poco más rezagados, la electricidad primaria (22.7\%) y el combustóleo $(7.2 \%)$.

Por su parte, en el grupo representativo de empresas semi-integradas el consumo energético total aumentó en $151 \%$ entre 1988 y 1994 , motivado por un incremento en la producción, de $126 \%$ en el mismo periodo. En especial, la electricidad primaria creció 146\%, aumentando su participación con relación al gas natural, el cual ha disminuido su contribución. Para 1994, el consumo se reportó de la siguiente manera: electricidad primaria (75.8\%), gas natural (9.3\%), combustóleo (8\%), y gas L.P. y diesel (6.9\%).

En la tabla 5 se muestran los valores para los poderes caloríficos empleados en la conversión de los datos notificados por las empresas, a petacalorías (10 ${ }^{15}$ calorías).

Tabla 5. Factores de conversión

\begin{tabular}{cccc}
\hline \hline Gas natural & $8460 \mathrm{kcal} / \mathrm{m}^{3}$ & Combustóleo & $10049.57 \mathrm{kcal} / \mathrm{t}$ \\
Coque & $6667920 \mathrm{kcal} / \mathrm{ton}$ & Gas LP & $1051320 \mathrm{kcal} / \mathrm{bl}$ \\
Electricidad & $860 \mathrm{kcal} / \mathrm{kWh}$ & Diesel & $1469600 \mathrm{kcal} / \mathrm{bl}$ \\
\hline \hline
\end{tabular}




\section{Fuentes endógenas}

Las principales fuentes endógenas de energía en una planta integrada con $\mathrm{AH} / \mathrm{CBO}$ ( $\mathrm{AH}$ : Alto Horno y $\mathrm{CBO}$ : Convertidor Básico al Oxígeno) son: el Gas de Coque (GC) de la carbonización del carbón; el Gas de Alto Horno (GAH) de la reducción del mineral de hierro con coque, y el Gas de escape de los convertidores al oxígeno o de Lingoteo y Desbaste (GLD) de la descarburación del arrabio líquido. Por otra parte, en las plantas integradas con RD/HEA (RD: Reducción Directa y HEA: Horno Eléctrico de Arco), el gas de escape de la reducción directa y del horno eléctrico de arco también pueden ser recirculados. Los subproductos gaseosos pueden ser usados como combustible para diversos hornos o para generar vapor y energía eléctrica.
En la encuesta del PUE se reportó el consumo de gas de coque y gas de alto horno en las empresas integradas entre 1988 y 1994 (tabla 6). Se observa que el consumo total de estos dos gases presentó un decremento de $14 \%$ en el periodo reportado, ocasionado por una disminución de 14.1 y $13.6 \%$ en el gas de alto horno y de coque, respectivamente. En 1994, el gas de alto horno abarcó $59 \%$ del consumo total, mientras que el de coque, el $41 \%$ restante. A su vez, este consumo total por fuentes endógenas representó $14.2 \%$ del total por fuentes exógenas en empresas integradas para el mismo año (tabla 6). Los datos de esta tabla nos indican que no se ha logrado una mejora en la eficiencia energética en cuanto a una mayor utilización de fuentes endógenas, por lo que representa una oportunidad para eficientar el consumo de energía en las empresas integradas.

Tabla 6. Consumo de energía por fuentes endógenas y exógenas en empresas integradas, 1988-1994

\begin{tabular}{|c|c|c|c|c|c|}
\hline \multirow{2}{*}{ Año } & \multicolumn{5}{|c|}{ Petacalorías } \\
\hline & $\begin{array}{c}\text { Gas de coque } \\
\mathrm{A}\end{array}$ & $\begin{array}{c}\text { Gas de alto horno } \\
\text { B }\end{array}$ & $\begin{array}{l}\text { Total de fuentes endógenas } \\
\qquad \mathrm{C}=\mathrm{A}+\mathrm{B}\end{array}$ & $\begin{array}{c}\text { Total de fuentes exógenas } \\
\text { D }\end{array}$ & $100(\mathrm{C} / \mathrm{D})$ \\
\hline 1988 & 3.465 & 4.992 & 8.458 & 45.377 & 18.6 \\
\hline 1989 & 3.358 & 4.534 & 7.892 & 45.132 & 17.5 \\
\hline 1990 & 3.496 & 4.759 & 8.255 & 49.503 & 16.7 \\
\hline 1991 & 3.074 & 4.232 & 7.305 & 45.051 & 16.2 \\
\hline 1992 & 2.963 & 4.051 & 7.015 & 45.042 & 15.6 \\
\hline 1993 & 2.844 & 3.985 & 6.829 & 47.633 & 14.3 \\
\hline 1994 & 2.992 & 4.286 & 7.278 & 51.163 & 14.2 \\
\hline
\end{tabular}

Fuente: encuesta del PUE

Los poderes caloríficos utilizados para convertir los datos notificados, a petacalorías fueron los siguientes: gas de coque $4200 \mathrm{kcal} / \mathrm{m}^{3}$, gas de alto horno $730 \mathrm{kcal} / \mathrm{m}^{3}$.

\section{b) Por proceso siderúrgico}

El consumo de energía primaria por procesos se muestra en la tabla 7. De igual forma, en la tabla 8 se muestran estos datos en términos de energía secundaria.
En la tabla 7 se distingue un descenso entre 1988 y 1991, debido principalmente al cierre de las instalaciones que usaban Horno Siemens Martin (HSM). A partir de 1992, año en que desapareció el HSM, el consumo por CBO se mantuvo casi constante, siguiendo a la producción. Para 1994, el consumo energético se distribuyó de la siguiente manera: en primer lugar el carbón primario, empleado en el alto horno, con $59 \%$, a continuación el gas natural $(22.3 \%)$ y finalmente, el combustóleo (13.4\%) y la electricidad primaria (5.3\%).

Tabla 7. Consumo de energía primaria (por proceso) en la industria siderúrgica, 1988-1994 En empresas que utilizan convertidor básico al oxígeno $(\mathrm{CBO})^{* *}$

\begin{tabular}{|c|c|c|c|c|c|c|}
\hline \multirow{2}{*}{ Año } & \multicolumn{6}{|c|}{ Petacalorías } \\
\hline & Combustóleo & Gas natural & Carbón primario & Electricidad primaria * & Otros 1 & Total \\
\hline 1988 & 4.211 & 6.068 & 17.909 & 2.056 & 0.602 & 30.847 \\
\hline 1989 & 3.939 & 5.810 & 16.028 & 2.120 & 0.587 & 28.483 \\
\hline 1990 & 4.232 & 6.090 & 17.259 & 2.456 & 0.980 & 31.016 \\
\hline 1991 & 3.471 & 5.857 & 14.311 & 1.996 & 0.052 & 25.688 \\
\hline 1992 & 3.469 & 6.208 & 14.719 & 1.828 & 0.003 & 26.227 \\
\hline 1993 & 3.948 & 6.361 & 14.606 & 1.356 & 0.000 & 26.272 \\
\hline 1994 & 3.662 & 6.078 & 16.071 & 1.453 & 0.000 & 27.263 \\
\hline
\end{tabular}


Tabla 7. Consumo de energía primaria (por proceso) en la industria siderúrgica, 1988-1994 (continuación) En empresas que utilizan horno eléctrico de arco (HEA)***

\begin{tabular}{|c|c|c|c|c|c|c|}
\hline \multirow{2}{*}{ Año } & \multicolumn{6}{|c|}{ Petacalorías } \\
\hline & Combustóleo & Gas natural & Carbón primario & Electricidad primaria * & Otros $^{1}$ & Total \\
\hline 1988 & 0.000 & 8.584 & 0.014 & 6.714 & 0.000 & 15.311 \\
\hline 1989 & 0.000 & 9.726 & 0.014 & 7.796 & 0.000 & 17.537 \\
\hline 1990 & 0.000 & 10.726 & 0.014 & 8.643 & 0.000 & 19.382 \\
\hline 1991 & 0.081 & 11.406 & 0.013 & 8.867 & 0.029 & 20.395 \\
\hline 1992 & 0.074 & 11.232 & 0.000 & 8.514 & 0.008 & 19.828 \\
\hline 1993 & 0.345 & 12.237 & 0.009 & 9.774 & 0.000 & 22.366 \\
\hline 1994 & 0.050 & 13.896 & 0.000 & 10.905 & 0.000 & 24.851 \\
\hline
\end{tabular}

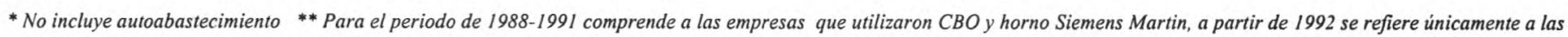
que emplearon $C B O$. *** Incluye tres empresas integradas y el grupo representativo de semi-integradas. 'Incluye gas LP y diesel Fuente: encuesta del PUE

Tabla 8. Consumo de energía secundaria (por proceso) en la industria siderúrgica, 1988-1994

En empresas que utilizan convertidor básico al oxígeno $(\mathrm{CBO}) * *$

\begin{tabular}{|c|c|c|c|c|c|c|}
\hline \multirow[b]{2}{*}{ Año } & \multicolumn{6}{|c|}{ Petacalorías } \\
\hline & Combustóleo & Gas natural & Coque & Electricidad* & Otros $^{1}$ & Total \\
\hline 1988 & 4.211 & 6.068 & 13.776 & 0.689 & 0.602 & 25.346 \\
\hline 1989 & 3.939 & 5.810 & 12.329 & 0.710 & 0.587 & 23.375 \\
\hline 1990 & 4.232 & 6.090 & 13.276 & 0.823 & 0.980 & 25.400 \\
\hline 1991 & 3.471 & 5.857 & 11.009 & 0.669 & 0.052 & 21.058 \\
\hline 1992 & 3.469 & 6.208 & 11.322 & 0.612 & 0.003 & 21.615 \\
\hline 1993 & 3.948 & 6.361 & 11.235 & 0.454 & 0.000 & 21.999 \\
\hline 1994 & 3.662 & 6.078 & 12.362 & 0.487 & 0.000 & 22.589 \\
\hline
\end{tabular}

En empresas que utilizaron horno eléctrico de arco (HEA)***

\begin{tabular}{|c|c|c|c|c|c|c|}
\hline \multirow{2}{*}{ Año } & \multicolumn{6}{|c|}{ Petacalorías } \\
\hline & Combustóleo & Gas natural & Coque & Electricidad* & Otros $^{1}$ & Total \\
\hline 1988 & 0.000 & 8.584 & 0.011 & 2.249 & 0.000 & 10.843 \\
\hline 1989 & 0.000 & 9.726 & 0.011 & 2.612 & 0.000 & 12.349 \\
\hline 1990 & 0.000 & 10.726 & 0.010 & 2.895 & 0.000 & 13.632 \\
\hline 1991 & 0.081 & 11.406 & 0.010 & 2.970 & 0.029 & 14.496 \\
\hline 1992 & 0.074 & 11.232 & 0.000 & 2.852 & 0.008 & 14.166 \\
\hline 1993 & 0.345 & 12.237 & 0.007 & 3.274 & 0.000 & 15.864 \\
\hline 1994 & 0.050 & 13.896 & 0.000 & 3.653 & 0.000 & 17.600 \\
\hline
\end{tabular}

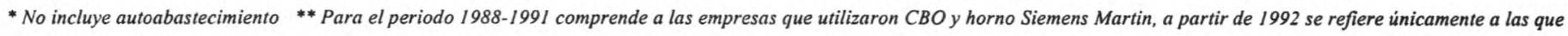
emplearon $C B O{ }^{* * *}$ Incluye tres empresas integradas y el grupo representativo de semi-integradas ${ }^{1}$ Incluye gas LP y diesel Fuente: encuesta del PUE 
Por otra parte, en el caso de las empresas que utilizaron HEA, el consumo energético presentó un incremento de $62.3 \%$ entre 1988 y 1994 (segunda parte de la tabla 7), provocado por un aumento de $82.3 \%$ en la producción. En 1994, el gas natural, empleado principalmente en la reducción directa del mineral, representó $56 \%$ del consumo; mientras que la electricidad primaria, utilizada fundamentalmente en el horno eléctrico, abarcó $43.9 \%$.

Por consiguiente, se puede concluir que las principales medidas para ahorrar energía en el sector deben ir encaminadas al proceso de reducción del mineral ( $\mathrm{AH}$ y $\mathrm{RD}$ ) y al horno eléctrico de arco, ya que en estas etapas del proceso se tienen altos consumos energéticos.

Consumo específico de energía en el sector

Un punto de comparación importante en materia energética es el relativo al Consumo Específico de Energía -CEE- (en la literatura especializada se maneja la abreviatura SEC por las iniciales en inglés de specific energy consumption).

En general, el CEE se define como la cantidad de energía primaria necesaria para realizar una determinada actividad, por ejemplo, la producción o procesamiento de un producto específico (Worrell et al, 1994). En el caso concreto de la industria siderúrgica, dicha actividad se refiere a la producción de una tonelada de un producto específico. El CEE se ve afectado por tres factores principales: los productos finales, el proceso de producción y la eficiencia del mismo. En cuanto al proceso de producción, éste puede ser descrito por sus entradas y salidas; a las primeras las constituyen las materias primas; las salidas se refieren al tipo y calidad de los productos. Por ejemplo, en cuestión de materias primas, el CEE depende de la calidad del carbón primario utilizado (porcentaje de cenizas) y, por otro lado, de la proporción de chatarra utilizada en el proceso de aceración.

El cálculo formal del CEE del sector se realiza de acuerdo con una metodología estándar prescrita por el Instituto Internacional del Hierro y del Acero (IISI por las iniciales en inglés de International Iron and Steel Institute), la cual toma en cuenta cada parte del proceso de producción de acero. Sin embargo, la metodología empleada en el cálculo del CEE de la industria siderúrgica nacional se refiere únicamente al cociente del consumo total de energía primaria entre la producción total de acero. Esto se debe a la falta de datos desagregados por cada parte del proceso de producción. De esta forma, en 1994 el CEE para la industria siderúrgica mexicana fue de 6361 millones de calorías (Mcal) por tonelada de acero (tabla 9).

Tabla 9. Consumo específico de energía (CEE) en la industria siderúrgica nacional, 1985-1994

\begin{tabular}{|c|c|c|c|c|c|c|c|c|c|c|}
\hline \multirow[b]{2}{*}{ Año } & \multicolumn{10}{|c|}{ Megacalorías / tonelada de acero } \\
\hline & 1985 & 1986 & 1987 & 1988 & 1989 & 1990 & 1991 & 1992 & 1993 & 1994 \\
\hline CEE & 8124 & 7396 & 7571 & 8833 & 8393 & 7772 & 7652 & 7146 & 6355 & 6361 \\
\hline
\end{tabular}

* Cifras preliminares Fuente: balances nacionales de energia, 1988-1994, Secretaria de Energía y CANACERO 1994

En los últimos años se registró una disminución sistemática del valor del CEE, lo cual muestra signos de eficiencia energética en el sector, ya que está produciendo más acero con un menor consumo energético. En este sentido, se pueden mencionar tres factores claves, que han contribuido al mejoramiento de la eficiencia energética: la gradual disminución y desaparición de los hornos Siemens Martin, la mayor utilización de la colada continua (tabla 10), y la disminución del consumo de coque por tonelada de arrabio en el alto horno (tabla 11).

Tabla 10. Grado de utilización de colada continua en la industria siderúrgica nacional, 1989-1993

\begin{tabular}{|c|c|c|c|c|c|}
\hline \multicolumn{6}{|c|}{ Grado de utilización de colada continua (porciento de la producción) } \\
\hline Año & 1989 & 1990 & 1991 & 1992 & 1993 \\
\hline Total nacional & 55.6 & 60.9 & 62.4 & 69.2 & 73.9 \\
\hline En empresas integradas & 52.9 & 58.2 & 58.8 & 65.9 & 71.9 \\
\hline En empresas semi-integradas & 68.5 & 74.8 & 79.5 & 85.3 & 83.2 \\
\hline
\end{tabular}

Fuente: CANACERO 1994 y encuesta del PUE

Tabla 11. Consumo específico de coque en la industria siderúrgica nacional

\begin{tabular}{cccccccc}
\hline \multicolumn{7}{c}{ Consumo específico de coque (kg / tonelada de arrabio) } \\
\hline Año & 1988 & 1989 & 1990 & 1991 & 1992 & 1993 & 1994 \\
Total nacional & 562 & 572 & 543 & 557 & 499 & 492 & 529 \\
\hline \hline
\end{tabular}


a) Por tipo de empresa

En la tabla 12 se observan los consumos específicos de energía en empresas integradas y en el grupo representativo de semi-integradas en el periodo 1988-1994, de acuerdo con los datos recabados en la encuesta del PUE.

Tabla 12. Consumo específico de energía en empresas integradas y en el grupo representativo de semi-integradas, 1988-1994

\begin{tabular}{ccccccccc}
\hline \multirow{2}{*}{ Año } & \multicolumn{7}{c}{ Megacalorías / tonelada de acero } \\
\cline { 2 - 7 } & 1988 & 1989 & 1990 & 1991 & 1992 & 1993 & 1994 \\
\hline \hline CEE Integradas & 7020 & 6966 & 6797 & 6873 & 6420 & 6333 & 6236 \\
CEE Semi integradas & 2609 & 3368 & 2898 & 3321 & 3219 & 2786 & 2750 \\
\hline
\end{tabular}

Fuente: encuesta del PUE

En el caso de las empresas integradas se ha registrado una tendencia a la baja, al pasar de 7020 a 6236 millones de calorías por tonelada ( $\mathrm{Mcal} / \mathrm{t}$ ) de acero en el periodo señalado. Este hecho está determinado principalmente por el comportamiento a la baja del CEE en las empresas que emplean HEA y por la mayor participación de este proceso a nivel nacional. Por otro lado, en el grupo representativo de semi-integradas, el CEE se ha mantenido entre 2600 y $3400 \mathrm{Mcal} / \mathrm{t}$ de acero.

Es conveniente señalar que el CEE obtenido en el grupo representativo de semi-integradas que emplean el proceso Chatarra-HEA es bajo en relación con el de las integradas, debido a que no contabiliza la energía del proceso de fabricación del hierro primario y la materia prima utilizada en el horno eléctrico es en su totalidad chatarra, la cual lleva un contenido energético. Esto último nos muestra la importancia que tiene en el CEE la materia prima empleada en la aceración.

\section{b) Por proceso siderúrgico}

El consumo específico de energía entre 1988 y 1994, por tipo de proceso en las empresas integradas se presenta en la tabla 13. Tanto las compañías que emplearon HEA como CBO en su proceso de aceración mostraron una tendencia decreciente en sus CEE, al pasar de 6354 a 5470 y de 7384 a $6413 \mathrm{Mcal} / \mathrm{t}$ de acero, respectivamente. En la literatura (PEMEX, 1985, por ejemplo), se menciona que el proceso $\mathrm{CBO}$ es más eficiente que el HEA; sin embargo, los datos de CEE reportados anteriormente señalan lo contrario. Esto se debe a que en su cálculo no se tomó en cuenta la proporción de chatarra utilizada en la etapa de aceración. Si se hubiera hecho esta consideración, seguramente el CEE relativo al CBO habría resultado menor.

\section{Comparaciones internacionales}

En primer lugar, se intenta comparar de una manera cualitativa el CEE de la industria siderúrgica mexicana con el de otros países. En este sentido, se remarca el hecho de que ésta no constituye una comparación formal y cuantitativa, ya que la metodología empleada en el cálculo del CEE en los otros países es diferente a la empleada en el caso de México. La utilidad de esta comparación estriba en ubicar cualitativamente el consumo energético del sector siderúrgico de nuestro país en el contexto internacional. Por lo que, en un estudio más profundo y más formal del CEE, los resultados obtenidos podrían diferir con los aquí presentados.

En segundo término, se comparan cuantitativamente los datos relativos al grado de utilización de colada continua, a la participación de la acería Siemens Martin, y al consumo específico de coque en el alto horno, en México y en otros países.

a)Comparación de consumos específicos de energía primaria

La figura 1 muestra los valores de CEE en diferentes países, incluyendo al nuestro, y el valor promedio en el mundo durante cuatro décadas comenzando en 1950. Para 1990, el CEE estimado en México fue de $7772 \mathrm{Mcal} / \mathrm{t}$, mucho más arriba de los valores reportados en los países desarrollados como Japón, Alemania Occidental y los Estados Unidos, que se situaron entre 4000 y $6000 \mathrm{Mcal} / \mathrm{t}$. Asimismo, se ubicó debajo del CEE de la India, el cual es alto debido a la baja calidad del carbón hindú (Bhaktavatsalam et al, 1995). Se observa también, que la disminución (gráficamente en forma de pendiente) del CEE en nuestro país ha sido más pronunciada en los últimos años que la presentada en los países desarrollados, lo cual parece indicar, que a partir de los años noventa, la eficiencia energética en la industria siderúrgica nacional se encuentra en una etapa similar a la que pasaron los países desarrollados y el promedio mundial en la década de los sesenta. Sin embargo, el sector del acero en México tiene todavía un camino largo que recorrer en los años venideros en lo que a eficiencia energética se refiere. 


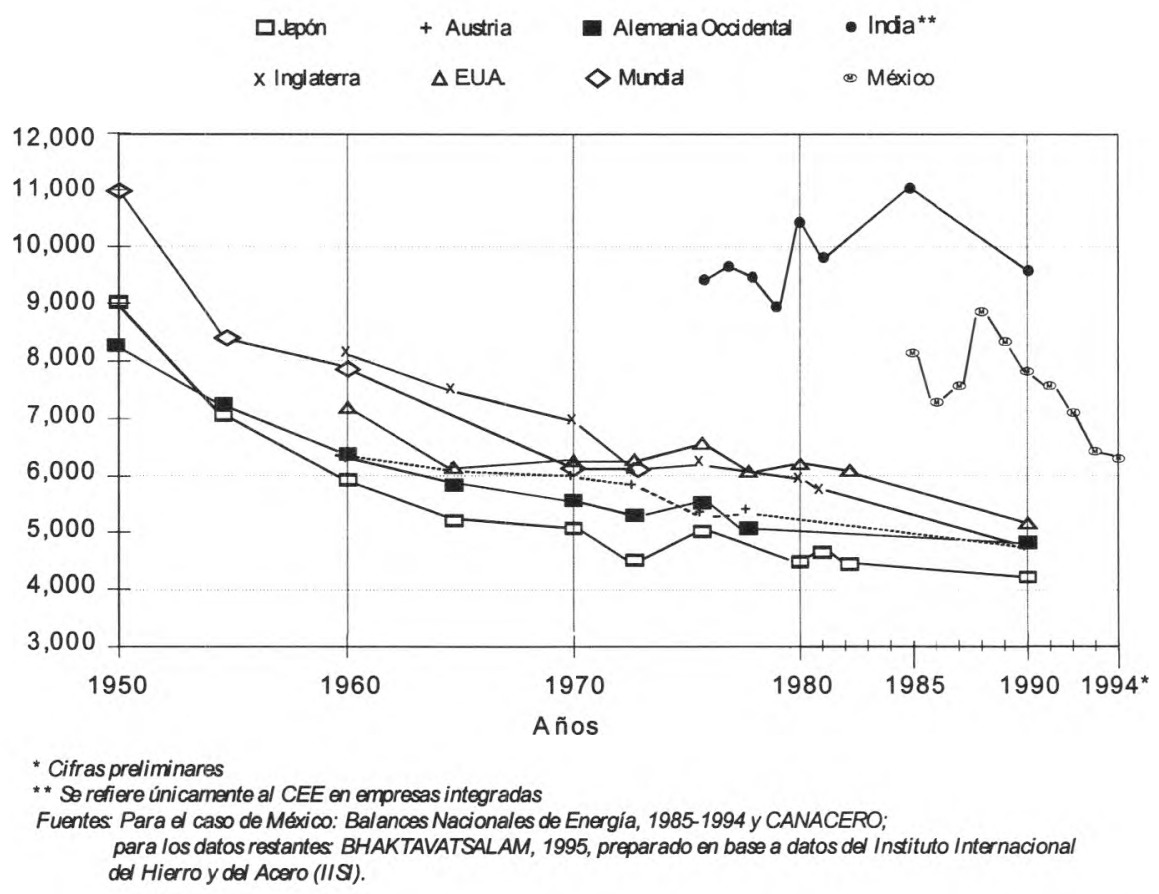

\section{Figura 1. Consumo especifico de energía en México, paises seleccionados y el mundo}

La dependencia del CEE de la utilización de HEA en el año 1988 en diferentes países de la Unión Europea se muestra en la figura 2. En el caso de los países europeos se incluye, además, un valor denominado mejor práctica. Para cada país, este valor se obtiene como el promedio pesado de los mejores consumos específicos por proceso detectados en Europa, por la fracción de la utilización del proceso en el país (Worrell et al, 1994). Por ejemplo, Holanda se encontró en un punto más cercano a su mejor práctica que España, lo que implica que aquel país alcanzó en ese año una mayor eficiencia energética que éste. En cuanto a México, no se ilustró su mejor práctica; no obstante, se puede decir que ésta debe situarse en el nivel de la de los países europeos. Entre 1988 y 1994, nuestro país redujo su CEE, acercándose así a su punto óptimo, aunque con un largo trecho que recorrer todavía.

También resulta interesante en la figura 2, el análisis de las cantidades marginales referentes, en primer lugar, a aquellos países que mostraron una proporción de HEA de cero por ciento, que comprendería al CEE del convertidor básico al oxígeno, dado que la participación del horno Siemens Martin es prácticamente nula (menos de uno por ciento). En esta situación, las cifras del sector mexicano con $\mathrm{CBO}$ se sitúan arriba de las de Luxemburgo, país que tiene bajo CEE debido al alto nivel de chatarra empleado en el CBO —-más de 30\% - (Worrell et $a l, 1994) ;$ y se sabe que $30 \%$ es la proporción óptima de chatarra/arrabio (UNIDO, 1990). En segundo lugar, se analizan las naciones con $100 \%$ de HEA, que al no considerar el proceso de reducción directa-horno eléctrico de arco, ya que su contribución en la producción de la Unión Europea es de 0.3\% (Worrell et al, 1994), nos reproduce la situación de las empresas semi-integradas (chatarra-HEA). En particular, los países europeos que se encuentran en esta situación presentaron altos CEE en relación con su mejor práctica, debido probablemente a la antigüedad de sus plantas y a los rápidos desarrollos tecnoló. gicos en el HEA. Por su parte, el grupo representativo de semiintegradas en México notificó valores de CEE inferiores a los europeos, que oscilan entre 2600 y $2800 \mathrm{Mcal} / \mathrm{t}$. Esto se debe, en gran medida, a que las plantas semi-integradas mexicanas son más recientes e involucran en sus procesos mayores avances tecnológicos; tal es el caso de la colada continua.

Tabla 13. Consumo específico de energía por proceso en empresas integradas, 1988-1994

\begin{tabular}{|c|c|c|c|c|c|c|c|}
\hline \multirow{2}{*}{ Proceso } & \multicolumn{7}{|c|}{ Megacalorías / tonelada de acero } \\
\hline & 1988 & 1989 & 1990 & 1991 & 1992 & 1993 & 1994 \\
\hline HEA & 6354 & 6182 & 6079 & 6112 & 5751 & 5663 & 5470 \\
\hline $\mathrm{CBO} *$ & 7384 & 7524 & 7312 & 7584 & 7005 & 7008 & 6413 \\
\hline
\end{tabular}


b) Otros factores de comparación

En la tabla 14 se muestran tres aspectos de comparación que tienen relación con la eficiencia energética en el sector: consumo específico de coque en el alto horno; participación del horno Siemens Martin y, finalmente, la proporción de colada continua en la producción total. Estas cifras complementan a las relativas de los consumos específicos de energía y así brindan una visión más amplia del sector siderúrgico nacional en el entorno de los países.

\section{Intensidad energética}

La intensidad energética en la industria siderúrgica mexicana se define como el consumo de energía primaria por unidad de Producto Interno Bruto (PIB) del sector.

En la tabla 15 se observa una caída de la intensidad energética en los últimos años, que reafirma el aumento de la eficiencia energética en el sector.

\section{Conclusiones}

La industria siderúrgica en nuestro país ha enfrentado un crecimiento de la demanda interna que, a pesar del incremento en la producción nacional, ha tenido que recurrir a importaciones durante los últimos años. Esto ha ocurrido en un periodo de relativo estancamiento en la economía. No obstante, la contracción de la demanda interna ocurrida por la crisis económica en 1995, se espera que la producción aumente debido a la expansión en el mercado de las exportaciones, motivada principalmente por la devaluación del Peso y la apertura comercial. En este sentido, el sector del acero - ahora privado - ha invertido grandes recursos para incrementar su capacidad de producción tanto de productos básicos como especializados. Las inversiones realizadas entre 1989 y 1994 totalizan 2500 millones de dólares.

Según datos de la CANACERO, para el periodo 1996-2000 se proyecta invertir unos 3700 millones de dólares, con lo que la capacidad instalada se ubicará en 17 millones de toneladas de acero al iniciar el nuevo siglo.

En años recientes se ha logrado una mejora en la eficiencia energética en la industria siderúrgica nacional, basada principalmente en aspectos tecnológicos y operativos, tales como la gradual disminución y desaparición de los hornos Siemens Martin, la mayor utilización de la colada continua y la disminución del consumo específico de coque en los altos hornos. Sin embargo, la industria del acero tiene todavía un largo camino que recorrer.

\section{Agradecimientos}

Los autores agradecen la asistencia y cooperación de la Dirección General de la Energía (DG XVII), Comisión Europea, Red COPED; la CANACERO, en particular del Lic. Miguel Elenes Inchaúrregui y el Ing. Manuel Gutiérrez Espinoza. Asimismo, a todas las empresas siderúrgicas que colaboraron con la encuesta del PUE, así como al personal del PUE quienes apoyaron la realización de la misma.

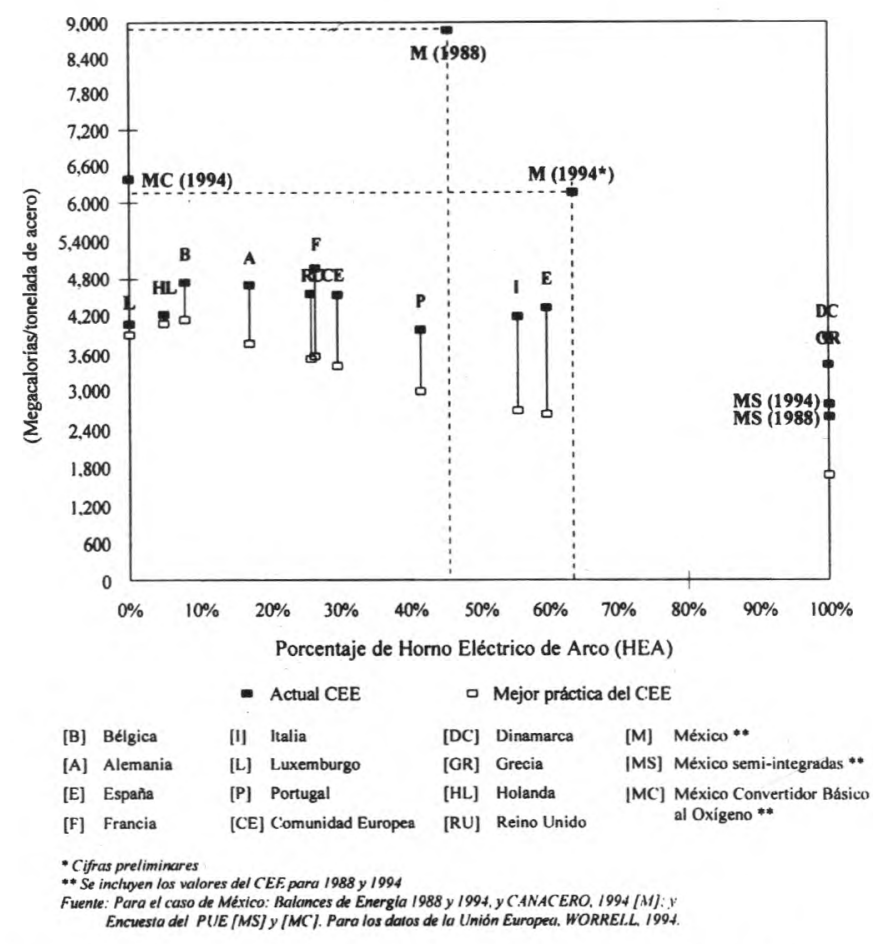


Tabla 14. Comparación de la eficiencia energética en países seleccionados

\begin{tabular}{|c|c|c|c|c|}
\hline \multirow{4}{*}{ País } & \multicolumn{2}{|c|}{$\begin{array}{l}\text { Consumo específico de coque } \\
\text { en el alto horno }\end{array}$} & $\begin{array}{l}\text { Participación del horno Siemens } \\
\text { Martin }\end{array}$ & $\begin{array}{l}\text { Grado de utilización de colada } \\
\text { continua }\end{array}$ \\
\hline & \multicolumn{2}{|c|}{$\mathrm{Kg} /$ ton de arrabio } & $\%$ de producción total & \multirow[t]{2}{*}{$\%$ de la producción total } \\
\hline & \multicolumn{3}{|c|}{ Año } & \\
\hline & 1980 & 1994 & 1991 & 1992 \\
\hline Japón & 449 & n.d & 0.0 & 95.4 \\
\hline Estados Unidos de América & 588 & n.d & 1.6 & 77.7 \\
\hline Italia & 463 & n.d & 0.0 & 97.7 \\
\hline Francia & 520 & n.d & 0.0 & 95.0 \\
\hline España & 530 & n.d & 0.0 & 94.0 \\
\hline China & 585 & n.d & n.d & 29.7 \\
\hline Brasil & 480 & n.d & 0.0 & n.d \\
\hline México & n.d. & 530 & 3.3 & 69.2 \\
\hline Turquía & 690 & n.d & 7.4 & n.d \\
\hline Portugal & 620 & n.d & 0.0 & n.d \\
\hline Argentina & n.d. & n.d & 0.0 & n.d \\
\hline Colombia & 740 & n.d & n.d & n.d \\
\hline
\end{tabular}

n.d.: no disponible Fuente: Siderurgia Latinoamericana (diciembre 1984, julio 1992 y mayo 1993) encuesta del PUE

Tabla 15. Intensidad energética en la industria siderúrgica nacional, 1985-1993

\begin{tabular}{|c|c|c|c|c|c|c|c|c|c|}
\hline \multirow[b]{2}{*}{ Año } & \multicolumn{9}{|c|}{ Intensidad energética (Kcal / \$ Peso 1980) } \\
\hline & 1985 & 1986 & 1987 & 1988 & 1989 & 1990 & 1991 & 1992 & $1993 *$ \\
\hline Total nacional & 1256 & 1209 & 1230 & 1378 & 1292 & 1211 & 1123 & 1107 & 1001 \\
\hline
\end{tabular}

* Cifras preliminares Fuente: JQM, preparado con base en los balances nacionales de energia, 1985-1993, SEMIP y a las cuentas nacionales, $1985-1993$

\section{Referencias}

Bhaktavatsalam, et al (1995). Specific Energy Consumption in the Steel Industry. Energy, Vol. 20, 1247-1995.

CANACERO (1994). Diez años de estadisticas siderúrgicas 1985-1994. Cámara Nacional de la Industria del Hierro y del Acero, México.

INEGI (1994). La industria siderúrgica en México 1994, Instituto Nacional de Estadística, Geografía e Informática, México.

PEMEX (1985). Consumo de energía en la industria siderúrgica. Subdirección de Planeación y Coordinación de Petróleos Mexicanos, México.

Ross (1987). Industrial Energy Conservation and the Steel Industry of the United States. Energy, Vol. 12, 1135-1987.

UNIDO (1990). United Nations Industrial Development Organization, Industry and Development Global Report 1990/1991.

Worrell et al (1994). Energy Consumption by Industrial Processes in the European Union. Energy, Vol. 19, 1113-1994.
Apéndice. Descripción de la encuesta dirigida por el PUE a la mayoría delas empresas del sector siderúrgico

Planteamiento de necesidades

El primer paso en el diseño del instrumento mediante el cual se evaluaría el sector comenzó con el reconocimiento de las principales variables que rigen al sistema siderúrgico, así como la consulta de trabajos de análisis precedentes tanto del propio (PUE), como de otras fuentes nacionales y extranjeras. Así pues, la definición de los principales parámetros que pudieran reflejar la situación real de la industria siderúrgica fue lo que más tarde determinaría el contenido de la encuesta. El proceso de determinación de necesidades tuvo una duración aproximada de dos semanas.

\section{Elaboración y elección del modelo}

Tras la elaboración de varios modelos, se optó por aquel que equilibrase la necesidad de un conocimiento pormenorizado 
de los diferentes procesos, con la factibilidad, desde el punto de vista de las empresas, de realizar labores internas de registro de datos, punto vital a la hora de calendarizar las diferentes etapas de desarrollo de la encuesta. Esta fase tuvo una duración aproximada de cuatro semanas.

\section{Aplicación}

La forma de aplicación fue individual, por empresa, comenzando por las situadas en el área metropolitana de la ciudad de México. 60 por ciento de los cuestionarios fue enviado por servicio de mensajería, junto con un breve folleto informativo de las actividades del PUE, así como un oficio de solicitud de la información en el cual se establecía el compromiso de presentar todos los datos recabados en forma agregada y no facilitarlos a terceras personas. El 40 por ciento restante fue enviado por Fax, junto con el citado oficio. Esta fase tuvo una duración aproximada de 14 semanas. Como se muestra en la tabla A1, 21 empresas en total respondieron la encuesta del PUE: las cinco integradas existentes en el país (en la tabla A1 se presentan seis, ya que HYLSA fue tomada como dos entidades debido a que los envíos a sus plantas de Puebla y Monterrey fueron independientes) y $16 \mathrm{de}$ las semi-integradas. De estas últimas, la tabla A2 destaca el número de empresas que proporcionaron información completa -datos de producción y de consumo de energía-. Con base en esta información se realizaron las tablas referidas al grupo representativo de empresas semi-integradas.

\section{Análisis de resultados}

El análisis de resultados se llevó a cabo a partir de la conformación de tablas anuales nacionales de producción y aspectos asociados, así como consumo energético. En apoyo de estas tablas se realizaron figuras sobre aspectos específicos. Las tablas fueron complementadas principalmente con datos de CANACERO (1994), además de algunos boletines publicados por esta misma institución, y el Balance nacional de energía de 1985 a 1994, publicados por SEMIP. Esta fase tuvo una duración aproximada de tres semanas.

Tabla A1

\begin{tabular}{|c|c|c|c|c|c|c|}
\hline \multirow{2}{*}{ Resultado de envío } & \multicolumn{2}{|c|}{ Empresas integradas } & \multicolumn{2}{|c|}{ Empresas semi-integradas } & \multicolumn{2}{|c|}{ Total } \\
\hline & número & $\%$ del total * & número & $\%$ del total $*$ & número & $\%$ de total $*$ \\
\hline respondió & 6 & 100 & 12 & 48.5 & 22 & 56.4 \\
\hline No respondió & 0 & 0 & 12 & 48.5 & 16 & 41.0 \\
\hline Otros ** & 0 & 0 & 1 & 3.0 & 1 & 2.6 \\
\hline
\end{tabular}

*Total de integradas o semi-integradas a las que se les envió el cuestionario, según el caso ** Incluye aquellas que no desearon responder la encuesta

Tabla A2

\begin{tabular}{cccccccc}
\hline \multirow{2}{*}{$\begin{array}{c}\text { Número total de empresas } \\
\text { semi-integradas con información } \\
\text { completa }\end{array}$} & 1988 & 1989 & 1990 & 1991 & 1992 & 1993 & 1994 \\
\cline { 2 - 7 } & 6 & 7 & 8 & 8 & 10 & 13 & 14 \\
\hline \hline
\end{tabular}

\section{Semblanza de los autores}

Alberto Elizalde Baltierra. Ingeniero mecánico electricista egresado de la Facultad de Ingeniería, UNAM en 1994 , y maestro en ingeniería (especialidad planeación) graduado en la misma Universidad en 1996. Actualmente se encuentra en Francia realizando estudios doctorales en economía industrial en la Universidad de París IX-Dauphine en cohabilitación con la Escuela Nacional Superior de Minas de París.

Juan García Peláez. Ingeniero mecánico electricista egresado de la Facultad de Ingeniería, UNAM en 1994. Ha participado en el desarrollo de diversos diagnósticos energéticos. Actualmente se encuentra laborando en una empresa farmacéutica de la iniciativa privada en el área de metrología y control.

Luis López Panadero. Ingresó a la Escuela Superior de Ingenieros Industriales de Madrid para cursar la carrera de ingeniería industrial, posteriormente vino a México e ingresa a la Universidad Iberoamericana para continuar su licenciatura. Finalizó sus estudios de ingeniería física y actualmente funge como director de desarrollo comercial de la firma Datasys de América.

Mariano Bauer Ephrussi. Investigador del Instituto de Física, UNAM. Cursó la licenciatura en física en la Facultad de Ciencias, UNAM (1951-1956) y obtuvo el doctorado en física en la Universidad de Maryland, EUA (1962). Su actividad profesional como investigador cubre fundamentalmente las áreas de física nuclear teórica, fundamentos de la mecánica cuántica, energía y medio ambiente.

Juan Quintanilla Martinez. Obtuvo la licenciatura en física en la Facultad de Ciencias, UNAM (1966), el doctorado en física con especialidad en física nuclear en la misma institución (1970) y posdoctorado en el Centro para la Física Teórica del Instituto Tecnológico de Massachussetts (1973-1974). Sus actividades de investigación se ubican en los campos de la física nuclear teórica, planificación científica y energética, así como energía y sus impactos ambientales. Actualmente, y desde 1982, funge como subdirector del Programa Universitario de Energía UNAM. 